\title{
Safety, tolerability and pharmacokinetics of intravenous ghrelin for cancer-related anorexia/cachexia: a randomised, placebo-controlled, double-blind, double-crossover study
}

\author{
F Strasser, , , TA Lutz ${ }^{2}$, MT Maeder ${ }^{3}$, B Thuerlimann ${ }^{4}$, D Bueche', M Tschöp ${ }^{5}$, K Kaufmann $^{2}$, B Holst ${ }^{6}$, \\ $M$ Brändle $^{7}, \mathbf{R}$ von Moos ${ }^{8}, \mathrm{R}_{\text {Demmer }}{ }^{4}$ and T Cerny ${ }^{4}$
}

'Oncological Palliative Medicine, Division Oncology/Haematology, Department Internal Medicine and Palliative Care Center, Department of IMD, Cantonal Hospital, Rorschacherstrasse, St Gallen 9007, Switzerland; ' ${ }^{2}$ Institute of Veterinary Physiology, Vetsuisse Faculty University of Zurich and Zurich Center of Integrative Human Physiology, Winterthurerstrasse 260, Zurich 8057, Switzerland; ${ }^{3}$ Division of Cardiology, University Hospital, Hebelstrasse 32, Basel 403I, Switzerland; ' Division Oncology/Haematology, Department Internal Medicine, Cantonal Hospital, Rorschacherstrasse, St Gallen 9007 , Switzerland; ${ }^{5}$ Department Psychiatry, Obesity Research Centre, University of Cincinnati - Genome Research Institute, 2180 East Galbraith Road, Cincinnati, OH 45237, USA; ${ }^{6}$ Laboratory for Molecular Pharmacology, The Panum Institute, University of Copenhagen, Blegdamsvej 3, Copenhagen DK-2200, Denmark; ' Division of Endocrinology and Diabetes, Department Internal Medicine, Cantonal Hospital, Rorschacherstrasse, St Gallen 9007, Switzerland; ${ }^{8}$ Division Oncology/Haematology, Department Internal Medicine, Cantonal Hospital, Loëstrasse 170, Chur 7000, Switzerland

Twenty-one adult patients were randomised to receive ghrelin on days I and 8 and placebo on days 4 and II or vice versa, given intravenously over a 60-min period before lunch: 10 received $2 \mu \mathrm{g} \mathrm{kg}^{-1}$ (lower-dose) ghrelin; II received $8 \mu \mathrm{g} \mathrm{kg}^{-1}$ (upper-dose) ghrelin. Active and total ghrelin, growth hormone $(\mathrm{GH})$, and insulin-like growth factor I levels were monitored at baseline (4-5 days before day I), during treatment days, and at end of study (day 17//8). Drug-related adverse events (assessed by NCl-CTC-toxicity criteria and cardiac examination) did not differ between ghrelin and placebo. No grade 3/4 toxicity or stimulation of tumour growth was observed. The peak increase of $\mathrm{GH}$, a biological marker of ghrelin action, was $25 \mathrm{ng} \mathrm{ml}^{-1}$ with lower-dose and $42 \mathrm{ng} \mathrm{ml}{ }^{-1}$ with upper-dose ghrelin. Morning fasting total ghrelin levels were higher $(P<0.05)$ for upper-dose patients at end of study $\left(3580 \mathrm{pg} \mathrm{ml^{-1 }}\right)$ than at baseline $\left(990 \mathrm{pg} \mathrm{ml}^{-1}\right)$. Insulin-like growth factor I levels did not change. At day 8, 81\% of patients preferred ghrelin to placebo as against $63 \%$ at the end of study. Nutritional intake and eating-related symptoms, measured to explore preliminary efficacy, did not differ between ghrelin and placebo. Ghrelin is well tolerated and safe in patients with advanced cancer. For safety, tolerance, and patients' preference for treatment, no difference was observed between the lower- and upper-dose group.

British Journal of Cancer (2008) 98, 300-308. doi: I0.1038/sj.bjc.6604I48 www.bjcancer.com

Published online 8 January 2008

(C) 2008 Cancer Research UK

Keywords: cachexia; anorexia; ghrelin; nutrition

Cancer patients often suffer from cancer anorexia/cachexia syndromes (CACS) and the consequences - fatigue, weakness, decreased performance status, poor tolerance of antineoplastic interventions, and psychosocial distress. Cancer anorexia/cachexia syndromes are characterised by a catabolic state triggered by tumour by-products, proinflammatory cytokines, and mediators of the neurohormonal system, causing loss of muscle and fat mass, anorexia, gastrointestinal dysmotility and early satiety, decreased anabolic drive, and hypermetabolism (Inui, 2002).

There are few pharmacological treatment options for CACS, but it has been shown that this patient population with advanced cancer has the capacity to respond to appetite stimulation therapy (Yavuzsen et al, 2005).

\footnotetext{
*Correspondence: Dr F Strasser; E-mails: florian.strasser@kssg.ch and fstrasser@bluewin.ch

This trial was registered at Current Controlled Trials: ISRCTN26I 85223. Received 13 August 2007; revised 2 November 2007; accepted 20 November 2007; published online 8 January 2008
}

Ghrelin, an endogenous ligand for the growth hormone (GH) secretagogue receptor, displays dose-dependent GH-releasing activity (Kojima et al, 1999). Ghrelin, which is predominantly secreted by gastric endocrine cells, stimulates food intake and triggers a positive energy balance through a central mechanism involving hypothalamic neuropeptides. In preclinical cachexia models, ghrelin has had stimulatory effects on appetite and food intake (Hanada et al, 2003; Wang et al, 2006), lean body mass (DeBoer et al, 2007), gastrointestinal motility (Date et al, 2002), energy metabolism, and proinflammatory cytokine expression (Dixit et al, 2004), and it has also alleviated cancer chemotherapy-associated dyspepsia (Liu et al, 2006) and vomiting (Rudd et al, 2006). These experimentally induced cancer models provide promising but not sufficient evidence for an effect of ghrelin in human cancer, prompting clinical studies in a representative clinical population, including long-term studies in humans.

In human volunteers, intravenous (i.v.) (Nagaya et al, 2001a; Wren et al, 2001; Akamizu et al, 2004; Schmid et al, 2005; Levin et al, 2006) or subcutaneous (Enomoto et al, 2003; 
Druce et al, 2006) ghrelin showed safety and tolerability at dosages up to $10 \mu \mathrm{g} \mathrm{kg}^{-1}$ - sufficient to promote orexigenic, prokinetic, and GH-releasing effects; in those studies, a sensation of warmth, sleepiness, bowel movements, and hunger were reported. Comparable results with i.v. ghrelin (single-dose bolus, daily for 3 weeks, or i.v. infusion) were reported in patients with chronic heart failure (Nagaya et al, 2001b, 2004), COPD (Nagaya et al, 2005), or diabetic gastroparesis (Murray et al, 2005).

In melanoma-bearing nude mice, ghrelin plasma concentration increased with cachexia progression (Hanada et al, 2004). In cancer patients with cachexia (various cancers, $n=21$ (Garcia et al, 2005); breast and colorectal cancer, $n=18$ (Wolf et al, 2006); lung cancer, $n=21$ (Shimizu et al, 2003)), ghrelin morning fasting levels were 1.3- to 1.5-fold higher than in those without cachexia and healthy controls. In contrast, ghrelin levels were normal in subgroups of patients in one study (39\%) (Wolf et al, 2006) and all patients in another study (gastric and colorectal cancer, $n=58$ ) (Huang et al, 2007). Lower ghrelin values in patients with colorectal cancer $(n=29)$ than in healthy controls $(n=50)$ were also reported (D'Onghia et al, 2007). Preliminary findings suggest that pharmacological doses of ghrelin alleviate cancer cachexia. Tumour-bearing mice showed improved food intake and body composition only at a high intraperitoneal dose $\left(40 \mu \mathrm{g} \mathrm{day}^{-1}\right)$ of ghrelin (Wang et al, 2006). In one pilot study, seven cancer patients had 31\% higher energy intake with i.v. ghrelin than with placebo $\left(5 \mathrm{pmol} \mathrm{kg}^{-1} \mathrm{~min}^{-1}\right.$ for $180 \mathrm{~min}$ equals $3 \mu \mathrm{g} \mathrm{kg}^{-1}$ ), with no adverse effects (Neary et al, 2004).

This trial was conducted to assess safety, tolerability, and pharmacokinetics in a 2-week trial of ghrelin infusion given intravenously, at one of two dose levels, once weekly, $1 \mathrm{~h}$ before lunch, to patients with far-advanced, incurable cancer, and involuntary loss of weight and appetite.

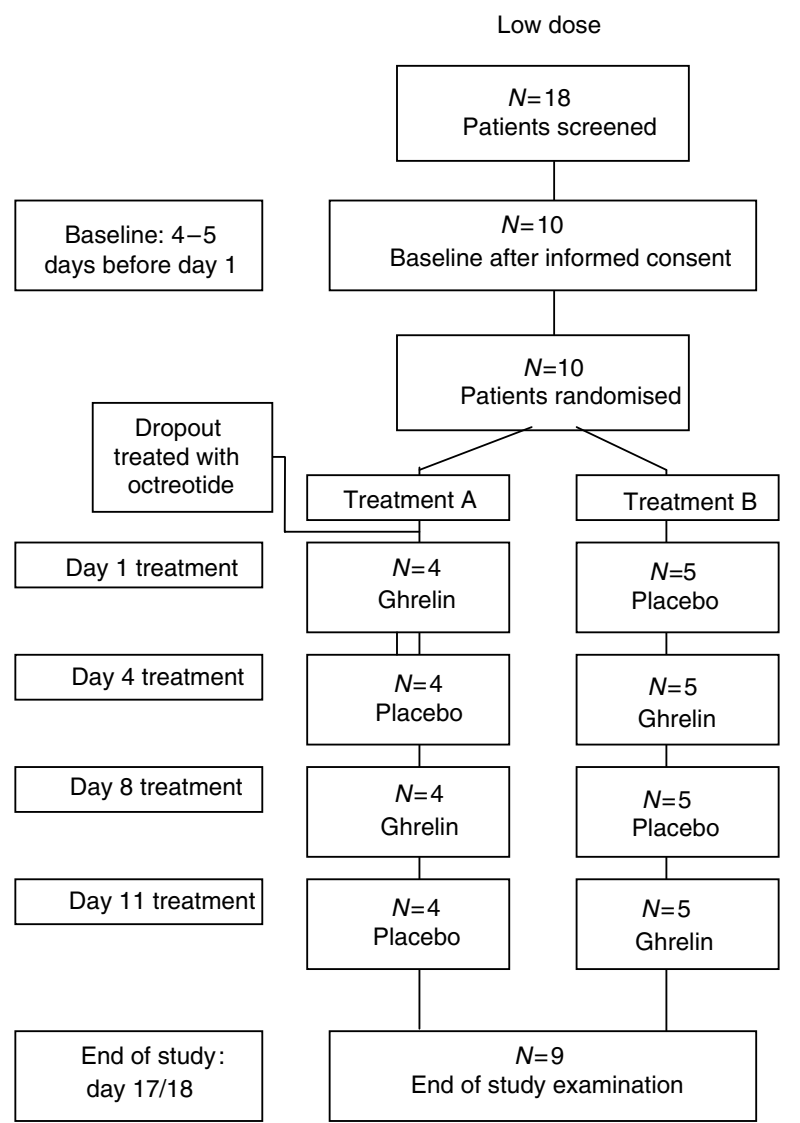

\section{MATERIALS AND METHODS}

This single-centre, randomised, double-blind, placebo-controlled, two-arm, double-crossover study adhered to Good Clinical Practice (GCP) and the Declaration of Helsinki and was approved by the local Ethical Review Board and Health Authorities.

\section{Participants}

Physicians at the Kantonsspital in St Gallen, Switzerland recruited adult patients with advanced incurable cancer who had loss of appetite ( $\geqslant 3$ visual analogue scale (VAS; $0=$ best, $10=$ worst)) and a weight loss of $\geqslant 2 \%$ within 2 or $\geqslant 5 \%$ within 6 months before the study not related to recent surgery.

Eligible patients gave written informed consent to participate, were able to eat without assistance, did not receive enteral or parenteral nutrition, and had no significant causes of secondary anorexia (defined as no severe symptoms or complications of the gastrointestinal tract impeding oral food intake) (Omlin and Strasser, 2007), as ensured by pre-baseline palliative oncology assessments. Patients were expected not to require new systemic antineoplastic treatment for the total study period of 3 weeks; those with unchanged continuous or weekly treatment for at least 2 months were eligible. Concomitant medication was to remain unchanged for at least 1 week before baseline. One patient having octreotide treatment was removed from analysis.

\section{Intervention}

In a randomised, double-blind, placebo-controlled, double-crossover trial, 4-5 days after baseline, patients received ghrelin on days 1 and 8 and placebo on days 4 and 11 or vice versa; end of study was day 17/18 (Figure 1).

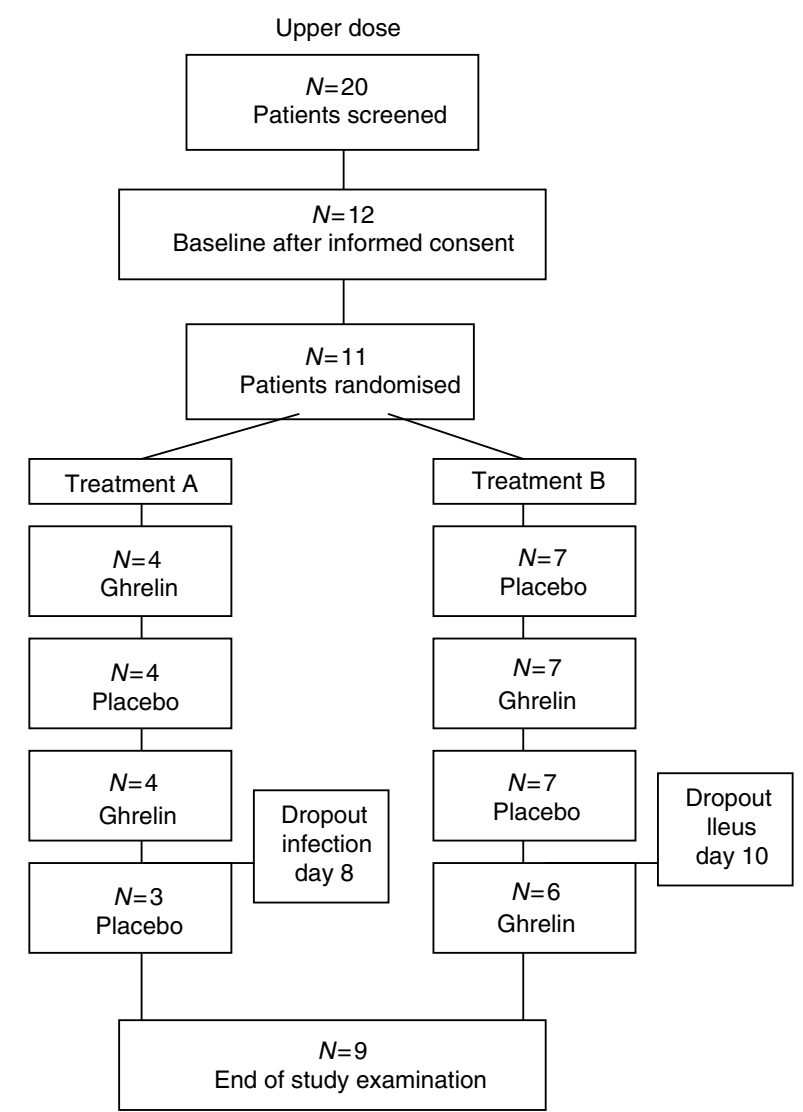

Figure I Flow of patients in a randomised, placebo-controlled, double-blind, double-crossover trial of i.v. ghrelin for cancer-related anorexia/cachexia. 
On treatment days, patients ingested only water from midnight to breakfast. In the outpatient clinic, safety laboratory values and fasting hormone blood samples (including testosterone in male patients) were drawn at 0800 hours; venous access was maintained for pharmacokinetics. Patients received a standardised breakfast ( $120 \mathrm{~g}$ bread, $20 \mathrm{~g}$ butter, $60 \mathrm{~g}$ jam, $200 \mathrm{ml}$ coffee with milk). At 1000 hours, a second i.v. line was inserted in the other arm for treatment given from 1030 to 1130 hours. Immediately after the end of the infusion, patients walked for approximately $5 \mathrm{~min}$ the $90 \mathrm{~m}$ to the restaurant where they received priority serving, starting the meal within $5 \mathrm{~min}$. A hospital volunteer accompanied the patients and documented the conditions (i.e., quality of service, quiet atmosphere) at lunch. Following evaluations performed after lunch, all patients, except one inpatient, returned home.

Ghrelin of Good Manufacturing Practice quality was purchased from Clinalfa (Merck Biosciences, Switzerland) as vials of $88 \mu \mathrm{g}$, stored at $-20^{\circ} \mathrm{C}$, and dissolved in $250 \mathrm{ml}$ normal saline by the hospital pharmacy immediately before application. The treatment was titrated up within the first $10 \mathrm{~min}$ ( $20 \%$ increase each $2 \mathrm{~min}$ ) and maintained for the next $50 \mathrm{~min}$. The lower-dose group (LD) received $10 \mathrm{pmol} \mathrm{kg}^{-1} \mathrm{~min}^{-1}$ (equals $0.0336 \mu \mathrm{g} \mathrm{kg}^{-1} \mathrm{~min}^{-1}$, approximately $2 \mu \mathrm{g} \mathrm{kg}^{-1}$ ). The dose was based on the reported maximal GH stimulation in human volunteers (Wren et al, 2001) and multiplied by 2 to account for suspected ghrelin resistance (Shimizu et al, 2003). After observing treatment tolerance in the LD patients, we administered the upper-dose group (UD) $40 \mathrm{pmol} \mathrm{kg}^{-1} \mathrm{~min}^{-1}$ (approximately $8 \mu \mathrm{g} \mathrm{kg}^{-1}$ ). Normal saline was used as placebo.

\section{Objectives}

We tested the safety and tolerability of two dose levels of i.v. ghrelin in patients with far-advanced cancer based on toxicity, tumour measurements, and patients' perceived tolerance. We also assessed the pharmacokinetics.

\section{Outcome measures}

Patients were assessed at each visit by using the NCI-CTC toxicity criteria Version 3.0, including standard blood examinations (hematology, chemistry panels), and cardiology evaluations, including echocardiography at baseline and at end of study. During the treatment phase, research personnel regularly contacted patients at home during the daytime to check for their safety. Before each infusion for each patient, the responsible investigator reviewed treatment logs for the preceding infusions and the results of the morning laboratory examinations. On study day 7 and at end of study, patients were asked about their perception of their tolerance and preference of day 1 (and 7) $v$ s day 4 (and 11) treatments. Radiological measurements were made within 2 weeks before the first infusion and within 2 weeks after the last infusion by CT-scans, except for two patients with liver metastasis who had once an ultrasound (no. 11) or an MRI (no. 18), for one patient monitored only by ultrasound (no. 11) or once at study end (no. 4), one patient who had a prior MRI liver metastasis monitored by ultrasound, and one patient who had an ultrasound of the liver. An independent radiologist reviewed all films made before baseline, at baseline, and at end of study to judge tumour size and dynamics.

Patients' nutritional intake was monitored daily. At baseline, dieticians assessed patients' food preferences, reviewed the procedures and meals for the next 2 weeks, and distributed a food scale and standard protocols for prospective 'third-person' (family members of patients) assessments (Bruera et al, 1986). A trained volunteer accompanied patients at lunch in a designated quiet section of the hospital restaurant. Meals were photographed and kitchen personnel documented the weight of each meal component before and after each patient ate it.
To detect acute symptom effects of treatment, VAS assessments $(0=$ best, $10=$ worst $)$ of appetite, hunger, anxiety, early satiety, nausea, and fatigue were measured before, during, and after infusion. Immediately after lunch on treatment days, VAS assessments of the pleasantness of the meal, perceived appetite, and perception of amount of food intake were obtained.

For ghrelin assays, $5 \mathrm{ml}$ blood was collected in a precooled EDTA vacutainer tube containing aprotinin ( $33 \mathrm{kIU})$, placed immediately on ice, and centrifuged $\left(4^{\circ} \mathrm{C}, 3000 \mathrm{G}, 5 \mathrm{~min}\right)$. For each millilitre of plasma, $10 \mu \mathrm{l}$ PMSF-Isopropanol, $50 \mu \mathrm{l} 1 \mathrm{~N}-\mathrm{HCL}$, and $50 \mu \mathrm{l}$ aprotinin were added, and aliquots were stored at $-80^{\circ} \mathrm{C}$ until batch analysis. Serum was collected and cooled for $\mathrm{GH}$, insulin-like growth factor 1 (IGF-1), interleukin-6 (IL-6), and leptin analysis. Testosterone radioimmunoassays (Diagnostic Products Corporation; Bühlmann, Salzburg, Austria) were performed from serum sent to routine safety labs. The radioimmunoassay kits used for total and active ghrelin were from Linco Research (St Charles, MO, USA); for GH (active GH IRMA immunoradiometric assay) from Diagnostic Systems Laboratory (Webster, TX, USA); for IGF-1 (human, bovine) from Peninsula Laboratories (San Carlos, CA, USA); for IL-6 (Quantikine human ELISA) from R\&D Systems (Minneapolis, MN, USA); and for leptin (human RIA) from Millipore (Billerica, MA, USA).

Autonomic dysfunction was assessed as described previously (Strasser et al, 2006). Standard deviation of beat-to-beat intervals (SDNN) was analysed at baseline and at end of study for $20 \mathrm{~min}$ in both the LD and UD; and in the UD, in addition, $30 \mathrm{~min}$ before infusion until after lunch on treatment days.

\section{Sample size}

Safety and tolerability was assessed on the basis of a sample size of 10 patients per dose level.

\section{Treatment assignment, randomisation, and blinding}

Patients were randomised by independent personnel at the hospital pharmacy, where the random allocation sequence produced (switches after 1 to maximal 3 patients) was assigned and the sealed envelopes for each patient distributed. A master randomisation list was kept in a locked container at the pharmacy. Copies of the documents in each sealed envelope were stored in a locked container accessible to clinicians for emergencies, as required by GCP standards.

Less than $30 \mathrm{~min}$ before each infusion, the pharmacy produced identical bags containing indistinguishable liquids of $250 \mathrm{ml}$ normal saline with or without ghrelin.

The database was closed after completion of the study and rating of all adverse events. Thereafter, an independent senior physician who had controlled the randomisation procedure, the master randomisation list, and the broken envelopes revealed the treatment assignments.

\section{Statistical analyses}

All analyses were performed with SPSS (Version 11.5). Descriptive statistics were used for demographic and baseline variables, frequencies of adverse events, and tumour measurements. For exploratory analyses of patients' preference of treatment, we used the exact binominal test. For pharmacokinetics (GH, ghrelin), glucose values, IGF-1 levels, nutritional intake, and SDNN, a comparison was made between changes from baseline for each individual subject between the two interventions (sum of two ghrelin periods $v s$ sum of two placebo periods). For betweenpatient comparison of peak GH (median), the Wilcoxon's (no-signed) rank sum test (Mann-Whitney) was used; and for within-patient comparisons (ghrelin morning fasting levels 3 days 
after the prior ghrelin infusion, glucose, IGF-1, nutritional intake, and SDNN) the Wilcoxon's signed rank test was used.

\section{RESULTS}

Flow (Figure 1) and demographics (Table 1) for the 20 patients studied were recorded. Oral intake at the fixed breakfast at treatment days was $296 \mathrm{kcal}$ (stable disease (SD) 80) in LD and $276 \mathrm{kcal}$ (118) in UD; one patient in LD and five in UD eat less than $250 \mathrm{kcal}$. Most patients (17 of 20) had ongoing inflammation (C-reactive protein (CRP) $>10 \mathrm{mg} \mathrm{ml}^{-1}$ ) (Fearon et al, 2006). Creatinine was $83 \mu \mathrm{moll}^{-1}$ (mean, SD 31) in LD and $73 \mu \mathrm{moll}^{-1}$ (17) in UD and one patient each in LD and UP had a value above normal $\left(<105 \mu \mathrm{moll}^{-1}\right)$. No patient had malignant gastroparesis. Two patients stopped study treatment early in the second week because of malignant bowel obstruction and blood-culture positive infection, respectively. Treatment of one patient was unblinded during the study because of apoplectiform deafness.

Concomitant pre-existing medications included laxatives (76\%), opioids $(67 \%)$, propulsive drugs $(67 \%)$, antacids $(62 \%)$, vitamins (57\%), and many others. Three patients (upper dose only) were on unchanged treatment for $>1$ week with megestrol acetate $(160 \mathrm{mg}$ twice daily) and three different patients on intramuscular testosterone, and one patient each received fish oil $(500 \mathrm{mg}$ twice daily) or dexamethasone $\left(8 \mathrm{mg} \mathrm{day}^{-1}\right)$. Six patients $(29 \%)$ received anticancer agents before and during the study (three gemcitabine; one each irinotecan, vinblastine, or bevacizumab). One patient was started on dexamethasone ( $4 \mathrm{mg}$ twice daily) for liver capsule pain on day 14.

Of 205 adverse events, 49 possibly and 9 probably were related to an agent studied - placebo as well as ghrelin (Table 2). They included abnormal liver tests or low potassium (three patients on ghrelin, four on placebo); increased serum amylase, creatinine, and D-dimer (seven on placebo); and increased CRP (two on ghrelin). Blinded clinicians rated the other adverse events as unrelated or probably unrelated to treatment. Those were cardiac arrhythmia during LD ghrelin infusion; constipation or infection with UD ghrelin; sinus tachycardia, pulmonary rales, increased stool frequency, or back pain with LD placebo; and blurred vision with UD placebo. Body temperature and oxygen saturation remained unchanged during and after the infusion of ghrelin and placebo in both dosage groups. Of 13 serious adverse events, one - transient apoplectiform deafness on day 13 - was judged as probably related to treatment on day 11 (placebo).

The mean scores for tolerability of the study medication immediately after infusion and after lunch did not differ between ghrelin and placebo or between LD and UD. More patients preferred ghrelin to placebo (Table 3) at day 7 and at end of study, with no evidence of patients' awareness of their treatment assignment.

During the study period, two patients experienced progressive disease (PD). Before enrolment, one had had SD and one, PD. Of 16 patients with SD during the study period, 10 had PD, five had $\mathrm{SD}$, and one had partial response before enrolment. Of two patients who stopped study treatment early, one had SD and one had PD at enrolment. The mean time interval between tumour assessments pre-baseline and at baseline was 79 days in LD and 29 days in UD, and between assessments at baseline and after the study was 34 and 25 days, respectively.

For total ghrelin, in the UD, elevated morning fasting levels were observed 3 days after the prior ghrelin infusion compared to after placebo $(P<0.001)$, as confirmed by an independent, blinded laboratory (Figure 2).

The mean differences of the peak GH levels (of week 1 and week 2) compared to baseline were higher in UD ( $50 \mathrm{ng} \mathrm{ml}^{-1}$ (SD 20)) than in $\mathrm{LD}\left(28 \mathrm{ng} \mathrm{ml}^{-1}(6)\right)(P=0.004)$.
Table I Demographics of 21 patients with cancer-related anorexia/ cachexia

\begin{tabular}{|c|c|c|}
\hline & $\begin{array}{l}\text { Lower-dose } \\
\text { group }(n=9)\end{array}$ & $\begin{array}{c}\text { Upper-dose } \\
\text { group }(n=I I)\end{array}$ \\
\hline \multicolumn{3}{|l|}{ Age (years) } \\
\hline Median (min, max) & $66(45,73)$ & $70(45,80)$ \\
\hline \multicolumn{3}{|l|}{ Gender } \\
\hline Female/male & $1 / 8$ & $2 / 9$ \\
\hline \multicolumn{3}{|l|}{ Diagnosis } \\
\hline Pancreatic cancer & I & 3 \\
\hline Mesothelioma & 2 & 0 \\
\hline Prostate cancer & 1 & 2 \\
\hline Colorectal cancer & 3 & I \\
\hline $\begin{array}{l}\text { Stomach/esophageal } \\
\text { cancer }\end{array}$ & 0 & 2 \\
\hline NSCLC & 1 & 2 \\
\hline Urogenital cancer & 1 & 0 \\
\hline Cholangiocarcinoma & 0 & I \\
\hline \multicolumn{3}{|c|}{ Metastasis (all patients $\geqslant 1$; none CNS) } \\
\hline Liver & 4 & 6 \\
\hline Lung & 4 & 2 \\
\hline Bone & 3 & 6 \\
\hline Peritoneal & 2 & 4 \\
\hline Lymph node & 1 & 8 \\
\hline \multicolumn{3}{|l|}{ Survival time (days) } \\
\hline Median (min, max) & $233(14,436)$ & $67(16,386)$ \\
\hline \multicolumn{3}{|c|}{ Prior chemotherapy (number of regimens) } \\
\hline 0 & 3 & 3 \\
\hline I & I & 4 \\
\hline 2 & 3 & 2 \\
\hline $3-5$ & 2 & 2 \\
\hline Prior radiation therapy & 3 & 5 \\
\hline Prior hormonal therapy & । & 2 \\
\hline \multicolumn{3}{|l|}{ Weight $(\mathrm{kg})$} \\
\hline Median (min, max) & $59(54,95)$ & $54(44,77)$ \\
\hline \multicolumn{3}{|l|}{ Body mass index $\left(\mathrm{kg} \mathrm{m}^{-2}\right)$} \\
\hline Median (min, max) & $21.7(15.7,30)$ & $20.6(17.3,30.4)$ \\
\hline \multicolumn{3}{|c|}{ Weight loss last 2 months (\%) } \\
\hline Median (min, max) & $3.6(2.2,15.1)$ & $6.0(3.6,11.5)^{\mathrm{a}}$ \\
\hline \multicolumn{3}{|c|}{ Nutritional intake at lunch (kcal) } \\
\hline Median (min, max) & $650(144,1133)$ & $304(179,700)$ \\
\hline \multicolumn{3}{|c|}{ Nutritional intake, whole day (kcal) } \\
\hline Median (min, max) & $1237(222,1864)$ & $889(179,1876)$ \\
\hline \multicolumn{3}{|c|}{ Appetite $(0=$ best, $10=$ worst $)$} \\
\hline Median (min, max) & $60(7,80)$ & $7 \mid(\mid 4,89)$ \\
\hline \multicolumn{3}{|c|}{ Early satiety $(0=$ no, $10=$ worst $)$} \\
\hline Median (min, max) & $25(2,79)$ & $52(10,95)$ \\
\hline \multicolumn{3}{|c|}{ Heart rate before Ist infusion (BPM) } \\
\hline Median (min, max) & $90(50,103)$ & $83(54,120)$ \\
\hline \multicolumn{3}{|c|}{ C-reactive protein $\left(\mathrm{mg} \mathrm{ml}^{-1}\right)$} \\
\hline Median (min, max) & $25(2,178)$ & $24(3,145)$ \\
\hline \multicolumn{3}{|c|}{ Free testosterone $\left(\mathrm{pmol}^{-1}\right)^{\mathrm{b}}$} \\
\hline Median (min, max) & $22.4(2.3,170)$ & $12.6(<0.5,25)$ \\
\hline \multicolumn{3}{|l|}{ Ghrelin, total (pg mll ${ }^{-1}$ ) } \\
\hline Median (min, max) & $104 \mid(317,1416)$ & $1015(533,2598)$ \\
\hline
\end{tabular}


Table I (Continued)

\begin{tabular}{|c|c|c|}
\hline & $\begin{array}{l}\text { Lower-dose } \\
\text { group }(n=9)\end{array}$ & $\begin{array}{c}\text { Upper-dose } \\
\text { group }(n=I I)\end{array}$ \\
\hline \multicolumn{3}{|l|}{ Ghrelin, active ( $\left.p g \mathrm{ml}^{-1}\right)$} \\
\hline Median (min, max) & $121(24,322)$ & $102(11,250)$ \\
\hline \multicolumn{3}{|l|}{ Leptin $\left(n g m l^{-1}\right)$} \\
\hline Median (min, max) & $0.8(0.4,6.4)$ & $1.9(0.3,7.4)$ \\
\hline \multicolumn{3}{|c|}{ Growth hormone $\left(\mathrm{pg} m l^{-1}\right)$} \\
\hline Median (min, max) & $1.2(0.3,4.9)$ & I $(0.3,3.8)$ \\
\hline \multicolumn{3}{|l|}{ IGF-I (pg per $100 \mu \mid)^{c}$} \\
\hline Median (min, max) & $3968(808,9 \mid 43)$ & $1820(\mid 121,3675)$ \\
\hline \multicolumn{3}{|c|}{ Plasma glucose $\left(\mathrm{mmoll}^{-1}\right)$} \\
\hline Median (min, max) & $4.8(4.1,7.5)$ & $5.6(4.4,7.9)$ \\
\hline \multicolumn{3}{|c|}{ Prior major gastrointestinal surgery } \\
\hline Gastrectomy & 0 & I \\
\hline Whipple procedure & 0 & i \\
\hline
\end{tabular}

$\mathrm{NSCLC}=$ non-small cell lung cancer; $\mathrm{CNS}=$ central nervous system; $\mathrm{BPM}=$ beats per minute; IGF-I = insulin-like growth factor I. ${ }^{a}$ Two patients had missing data on weight loss 2 months before study entry, but had weight loss 6 months before study

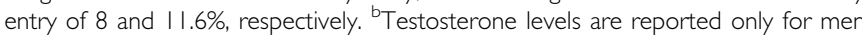
$\left(\mathrm{LD} n=8, \mathrm{UD} n=9\right.$ ). ${ }^{9}$ GF- $\mid$ levels are reported for $\mathrm{LD} n=8$ and UD $n=11$.

Table 2 Adverse events of treatment with intravenous ghrelin in 21 patients with cancer-related anorexia/cachexia

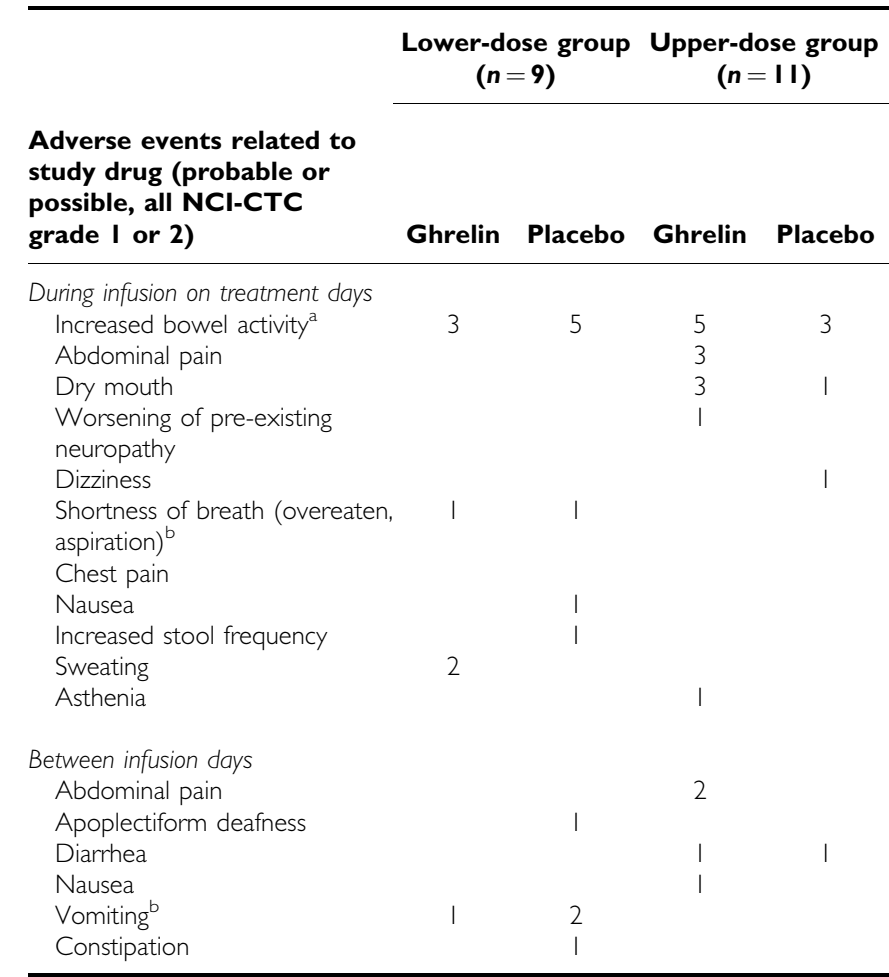

$\mathrm{NCl}-\mathrm{CTC}=$ common toxicity criteria $(\mathrm{CTC})$ established by the National Cancer Institute (NCl). ${ }^{a}$ In five patients both on ghrelin and placebo. ${ }^{b}$ In one patient both on ghrelin and placebo.

In one patient (UD) having gastrectomy 6 months before baseline, neither substantial differences in baseline values nor peak levels of active or total ghrelin or GH were detected.
Insulin-like growth factor 1 did not increase at day $17 / 18$ as compared to study start in any patient examined (maximal increase from baseline was $170 \%$ ); mean IGF-1 was 1359 pg per $100 \mu \mathrm{l}( \pm 994)$ in $\mathrm{LD}(n=7)$ and $1096 \mathrm{pg}$ per $100 \mu \mathrm{l}( \pm 495)$ in UD $(n=9)$, and mean change from baseline $-2624 \mathrm{pg}$ per $100 \mu \mathrm{l}$ $( \pm 2888)$ and -624 pg per $100 \mu \mathrm{l}( \pm 962)(P=0.055)$.

During treatment days, blood glucose values compared to baseline after infusions were higher when patients received ghrelin than when receiving placebo in LD only after lunch (3.6 vs $\left.2.5 \mathrm{mmoll}^{-1}(P=0.005)\right)$ but not after infusion $(1.5$ vs $\left.1.3 \mathrm{mmoll}^{-1}, P=0.16\right)$, in UD both after lunch $\left(2.4 v s 1.3 \mathrm{mmol}^{-1}\right.$ $(P=0.01))$ and after infusion $\left(0.8 v s 0.2 \mathrm{mmoll}^{-1}(P=0.044)\right)$.

Plasma levels of IL-6 did not change throughout the treatment period (results not shown).

There were no significant differences in nutritional intake or symptoms compared to baseline when patients received ghrelin or placebo. Nutritional-intake-at-lunch compared to baseline was in LD $-105 \mathrm{kcal}$ with ghrelin and $-17 \mathrm{kcal}$ with placebo, in UD 251 and $230 \mathrm{kcal}$, respectively; nutritional-intake-at-lunch-and-rest-ofthe-day was (LD) 145 and $228 \mathrm{kcal}$, and (UD) 244 and $156 \mathrm{kcal}$, respectively (all $P=\mathrm{NS}$ ). In UD patients not receiving concurrent chemotherapy $(n=8)$, a trend towards increased differences compared to baseline for nutritional-intake-at-lunch-and-rest-ofthe-day (ghrelin: $448 \mathrm{kcal}$; placebo: $128 \mathrm{kcal} ; P=0.093$ ) but not nutritional-intake-at-lunch (ghrelin: $330 \mathrm{kcal}$; placebo: $200 \mathrm{kcal}$; $P=\mathrm{ns}$ ) was observed.

Mean SDNN was $57 \pm 28 \mathrm{~ms}$ at baseline and $73 \pm 57 \mathrm{~ms}$ at end of study in 18 evaluable patients $(P=\mathrm{ns})$; in UD $(n=9)$, for ghrelin $84 \pm 40 \mathrm{~ms}$ and for placebo $78 \pm 35 \mathrm{~ms}$ in week $1(P=\mathrm{NS})$, and in week $2,75 \pm 35$ and $80 \pm 27 \mathrm{~ms}(P=\mathrm{NS})$, respectively.

\section{DISCUSSION}

This is, to our knowledge, the first trial investigating two doses of ghrelin in patients with advanced cancer and anorexia/cachexia. Intravenous ghrelin infusion for $60 \mathrm{~min}$ at 2 or $8 \mu \mathrm{g} \mathrm{kg}^{-1}$ body weight is well tolerated and safe in these patients who represent a 'real world' population of cancer patients with anorexia/cachexia.

At present, no dose-limiting toxicity has been reported for ghrelin in animals or humans. The dosage used was based on the reported maximal GH stimulation in human volunteers (Wren et $a l, 2001)$ and on prior trials using up to $10 \mu \mathrm{g} \mathrm{kg}^{-1}$ in healthy volunteers and $6 \mu \mathrm{g} \mathrm{kg}^{-1}$ in patients (Nagaya et al, 2001b). Our data suggest a dose relationship with $\mathrm{GH}$ stimulation. In cachectic tumour-bearing mice (MCG101), higher ghrelin doses were required to increase food intake and body weight than in control mice (Wang et al, 2006). Other interventional CACS studies did not compare ghrelin doses (Hanada et al, 2003; Neary et al, 2004; DeBoer et al, 2007).

Morning fasting levels of ghrelin in patients (Shimizu et al, 2003; Garcia et al, 2005; Wolf et al, 2006; Huang et al, 2007) or animals (Hanada et al, 2004; Liu et al, 2006) with CACS are still poorly understood. In animals, both higher (Hanada et al, 2004) and lower (Liu et al, 2006) ghrelin levels than controls are reported. Several studies showed higher ghrelin levels (Shimizu et al, 2003; Garcia et al, 2005) or higher levels only in subgroups (61\% of 18 breast and colorectal cancer patients) (Wolf et al, 2006) in patients with CACS as compared to non-cachetic cancer patients or healthy controls; however, normal (Huang et al, 2007) ghrelin levels were reported as well. The differences of fasting levels of ghrelin in cancer patients may be explained by differences in BMIs, $20.7 \mathrm{~kg} \mathrm{~m}^{-2}$ in LD and $20.6 \mathrm{~kg} \mathrm{~m}^{-2}$ in UD, and $18.5 \mathrm{~kg} \mathrm{~m}^{-2}$ in others (Shimizu et al, 2003). It remains unclear whether ghrelin plasma levels are increased in cancer patients and whether high plasma levels of ghrelin will induce resistance to ghrelin. It remains to be clearly shown whether the response to peripheral ghrelin differs depending on the prevailing ghrelin level. 
Table 3 Patients' preference of treatment between placebo and intravenous ghrelin

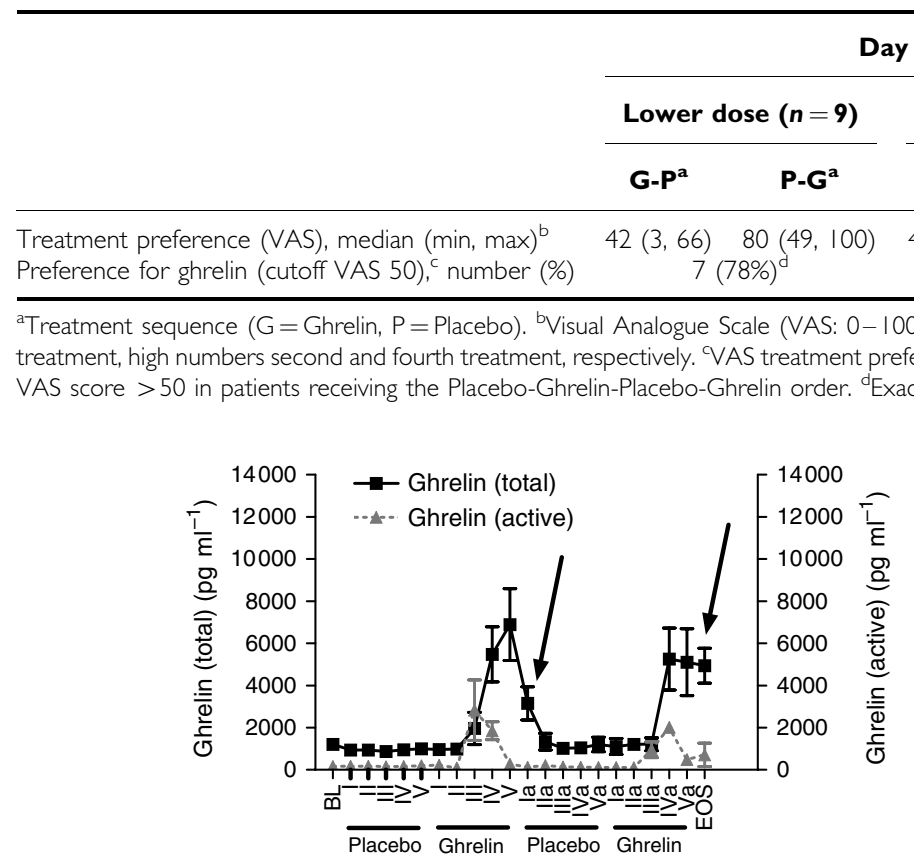

$$
\text { Day } 7
$$

End-of-study

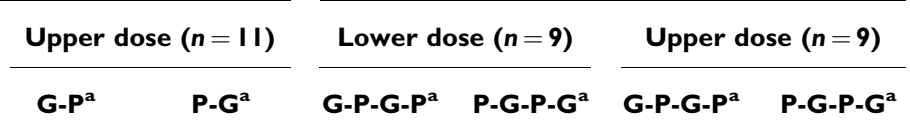

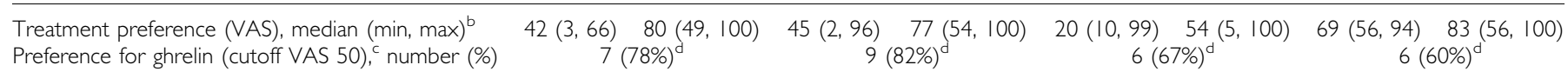

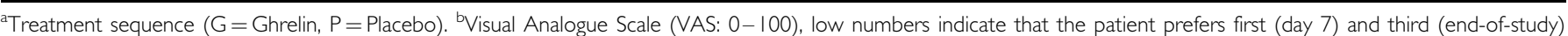
treatment, high numbers second and fourth treatment, respectively. ${ }^{\complement}$ VAS treatment preference score $<50$ in patients receiving the Ghrelin-Placebo-Ghrelin-Placebo order, I00VAS score $>50$ in patients receiving the Placebo-Ghrelin-Placebo-Ghrelin order. ${ }^{d}$ Exact binominal two-sided tests: 4: $P=N S, 5: P=0.065,6: P=N S$.

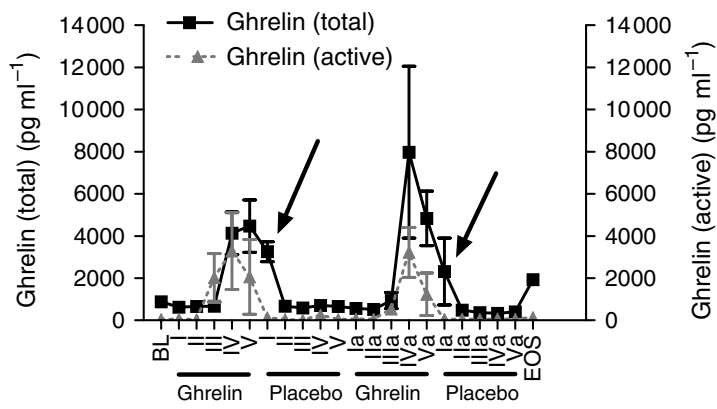

Figure 2 Pharmacokinetics of active and total ghrelin of the upper dose patients. BL, baseline; I-V, blood samples week I (I: morning fasting; II: immediately before ghrelin infusion ( I 030); III: during ghrelin infusion; IV: after ghrelin infusion (I I 30); V: after lunch ( I 230)); la-Va, blood samples week 2; EOS, end of study. $P<0.00$ I for differences of morning fasting level of total ghrelin 3 days after ghrelin or placebo.

In transgenic mice overexpressing ghrelin, the acute stimulation of food intake of exogenous ghrelin was not diminished (Wei et al, 2006). In patients with anorexia nervosa, in whom chronic hyperghrelinaemia presents with two- to three-fold increased levels (Broglio et al, 2004), i.v. ghrelin $\left(1 \mu \mathrm{g} \mathrm{kg}^{-1}\right.$ per hour for $5 \mathrm{~h}\left(5 \mathrm{pmol} \mathrm{kg}^{-1} \mathrm{~min}^{-1} \times 300 \mathrm{~min}\right.$; Miljic et al, 2006) or $1 \mu \mathrm{g} \mathrm{kg}^{-1}$ (Broglio et al, 2004)) caused much lower GH and glucose increases than in healthy volunteers and caused no appetite stimulation but increased sleepiness. In two patients having ghrelin-producing tumours in gastro-entero-pancreatic system (Corbetta et al, 2003; Tsolakis et al, 2004), BMI remained high and the appetite good despite failure to respond to anticancer treatment.

In our study, we observed no major unexpected tumour-growth dynamics, but the study design with short intervals of the tumour measurements impedes firm conclusions. As ghrelin is also a potent GH secretagogue, there are concerns about GH-mediated stimulation of tumour growth, especially regarding treatment of cancer patients. Several cell lines express the ghrelin receptor (Yeh et al, 2005; Ekeblad et al, 2006) and secrete ghrelin (Yeh et al, 2005). In vitro studies suggest that ghrelin may enhance the proliferation of prostate (Yeh et al, 2005) and pancreatic (Duxbury et al, 2003) cancer cells, but not of a lung cancer cell line, where it induced dose-dependent inhibition of cell proliferation and increased apoptosis (Cassoni et al, 2006). Some tumours from archival samples express ghrelin (Jeffery et al, 2005), whereas others do not (Cassoni et al, 2006; Mottershead et al, 2007). Tumour incidence is not increased in patients with anorexia nervosa (Mellemkjaer et al, 2001) despite elevated ghrelin levels. The hepatic GH effector IGF-1 levels are not correspondingly increased in conditions with high endogenous plasma ghrelin levels, such as ghrelin-producing tumours (Corbetta et al, 2003; Tsolakis et al, 2004). Furthermore, the administration of ghrelin does not significantly affect the IGF-1 level in healthy volunteers (Enomoto et al, 2003), patients with cardiovascular (Nagaya et al, 2004) or pulmonary diseases (Nagaya et al, 2005), tumour-bearing animals (DeBoer et al, 2007), or our patients with CACS. In contrast, studies using synthetic oral ghrelin mimetics have shown a significant effect on the IGF-1 level in volunteers and in the frail elderly (Smith, 2005) or patients with cancer cachexia (Garcia et al, 2007).

Higher morning fasting total ghrelin levels 3 days after i.v. ghrelin administration suggest a carryover effect. As the half-life of ghrelin is short - approximately $15 \mathrm{~min}$ - a systematic mistake in the analysis was thought likely, but an independent, blinded laboratory confirmed our results. Ghrelin levels were normal before infusion at 1030 hours. Renal function was not impaired. These unexpected findings of total, but not active, ghrelin remain unexplained at present but may indeed be without any physiological significance.

Our study of patients with far-advanced cancer was not designed to detect effects on nutritional intake, eating-associated symptoms, or lean-body mass. We found no major differences for these efficacy parameters between ghrelin and placebo in preliminary analyses. Our finding contrasts with the data observed in animal models (Hanada et al, 2003; Wang et al, 2006; DeBoer et al, 2007). Our methodology with treatment of secondary anorexia, nutritionist-monitored lunch meals, full placebo control of all outcomes, and standardised procedures and time schedules makes systemic errors unlikely.

In contrast to one recent small series of patients with mainly (5/7) breast cancer (Neary et al, 2004), our patients had tumours typically leading to CACS. A high intrapatient variability of symptoms and nutritional intake is reported in patients with advanced, incurable cancer (Stromgren et al, 2006). Baseline food intake (Gilg and Lutz, 2006) and dietary patterns with high protein or liquid intake (Blom et al, 2006) may influence ghrelin regulation. Drugs for symptom control (eg, haloperidol (Jaszberenyi et al, 2006), 5-HT-3 antagonist (Depoortere et al, 2006)) may interact with ghrelin metabolism. Patients often have enhanced levels of proinflammatory cytokines and stress, which are reported to increase preprandial activation of ghrelin secretion (Kristenssson et al, 2006) by activation of sympathetic nerves but not by epinephrine (Mundinger et al, 2006). Alterations in testosterone 
levels may influence ghrelin regulation, as testosterone treatment in prepubertal boys decreased ghrelin values (Lebenthal et al, 2006). Patients may have remaining side effects of prior chemotherapy mediating CACS (Hutton et al, 2007).

Ghrelin may prolong the premature gastric phase III of migrating motor complex tone in the proximal stomach (Tack et al, 2006), leading to enhanced gastrointestinal motility without (Tack et al, 2006) and with (Liu et al, 2006) increased food intake, but some studies show no stimulatory effect of ghrelin on motility (Depoortere et al, 2006; Ohno et al, 2006). Patients with advanced cancer often have autonomic dysfunction (Strasser et al, 2006), as did $83 \%$ of our patients. We found no differences in autonomic function during or after two single infusions of ghrelin.

Some of our patients seem to prefer ghrelin to placebo, and this may be associated with the effect of peripheral ghrelin targeting the mesolimbic reward circuitry (Abizaid et al, 2006). The limitations of this study design include the lack of chronic efficacy data beyond two weekly infusions; namely, body composition (lean body mass, fat mass (Theander-Carrillo et al, 2006)), objective subconscious locomotive motor and physical activity (Jaszberenyi et $a l, 2006$ ), energy expenditure measurements (Lejeune et al, 2006), and gastrointestinal motility (Binn et al, 2006; Blom et al, 2006). Our results on dose responsiveness may be influenced by unbalanced groups: UD patients had more metastases, greater weight loss, lower dietary intake, more early satiety, and were closer to death. However, given the double-crossover design, no effect on outcomes is expected from unequal (4vs 7) randomisation in the UD group.

The patients in our study represent a very diverse population, since this trial was conducted with more or less unselected patients, with CACS having mostly far advanced cancer, as the median survival documents, and various tumour types. Our main aim was to explore the safety and tolerability of i.v. ghrelin in such a clinical situation, in which the interpretation of (negative) efficacy data requires considerable caution. In the two patients with stomach and oesophageal cancer, the clinical efficacy of ghrelin may be limited, since in patients having had vagotomy ghrelin induced only an increase in GH secretion but not in energy intake (le Roux et al, 2005). Given the foreseen clinical application, namely a relatively short interval between intervention and meal but not at the same time (difficult for patients to have continuous infusions during meals or to inject subcutaneously 'real time' during meals), we chose to offer lunch immediately after, but not during, the ghrelin infusion, and this time difference may explain the lack of difference in energy intake observed between ghrelin and saline. The safety and tolerability data support further exploration of the therapeutic potential of natural ghrelin, namely escalation of dose (Wang et al, 2006) and frequency and chronic administration. The patient population may be stratified for baseline ghrelin levels (Garcia et al, 2005; Wolf et al, 2006), and other factors need to be controlled for, namely genetic alterations of the ghrelin gene (Holst and Schwartz, 2006), cytokine levels (Dixit et al, 2004), stress level (Kristenssson et al, 2006), hypogonadism (Strasser et al, 2006), patients' eating preferences (Blom et al, 2006), baseline food intake (Gilg and Lutz, 2006), and gastric emptying (Binn et al, 2006). These strategies may counteract the series of many negative cachexia phase III trials (EPA, cannabinoids) or single not confirmed studies (ATP, thalidomide), treating uniformly all patients having loss of weight and appetite, despite promising pathophysiological concepts.

In conclusion, ghrelin administered intravenously as one therapeutic dose and repeated once after 1 week was safe and well tolerated by both LD and UD patients with far-advanced cancer and anorexia/cachexia. Several patients preferred ghrelin to placebo despite a lack of major differences in food intake or symptoms. The stimulation of $\mathrm{GH}$, reflecting biological activity, was dose-dependent. Anorexia/cachexia remains a burdensome clinical problem with few treatment options. Further research with ghrelin will explore dose escalations, route and schedule modifications, and mechanisms of ghrelin resistance.

\section{ACKNOWLEDGEMENTS}

We thank our patients for engaged participation, the nursing staff of the oncology outpatient unit for invaluable support, and the oncologists who referred patients to the study. Countless technicians, laboratory professionals, and the oncology research unit supported this trial, including Dr S Thammacharoen from the Institute of Veterinary Physiology, Monika Diethelm from the body composition unit, Doris Giselbrecht et al from the nutritionist team, Monika Ott from cardiology, Beppi Osterwalder as the independent senior physician performing the unblinding of the database, and the independent radiologists who reviewed all films to judge tumour size and dynamics. Tina Nielsen and Hans Schambye from GastroTech Pharm A/S, Denmark, assisted in data presentation blinded to treatment assignment. Shu-Fang HsuSchmitz provided statistical advice. Scientific editing was provided by Susan Eastwood ELS(D). Research support was provided by OncoSuisse OCS - 01385 - 08 - 2003: scientific project grant; Eastern Switzerland Cancer Research Fund: scientific project support; Swiss Institute of Applied Cancer Research: pilot development grant; Gastrotech Pharm A/S, Denmark: unrestricted research support; and Amgen Switzerland: unrestricted grant.

\section{REFERENCES}

Abizaid A, Liu ZW, Andrews ZB, Shanabrough M, Borok E, Elsworth JD, Roth RH, Sleeman MW, Picciotto MR, Tschop MH, Gao XB, Horvath TL (2006) Ghrelin modulates the activity and synaptic input organization of midbrain dopamine neurons while promoting appetite. J Clin Invest 116: $3229-3239$

Akamizu T, Takaya K, Irako T, Hosoda H, Teramukai S, Matsuyama A, Tada H, Miura K, Shimizu A, Fukushima M, Yokode M, Tanaka K, Kangawa K (2004) Pharmacokinetics, safety, and endocrine and appetite effects of ghrelin administration in young healthy subjects. Eur J Endocrinol 150: $447-455$

Binn M, Albert C, Gougeon A, Maerki H, Coulie B, Lemoyne M, Rabasa Lhoret R, Tomasetto C, Poitras P (2006) Ghrelin gastrokinetic action in patients with neurogenic gastroparesis. Peptides 27: 1603-1606

Blom WA, Lluch A, Stafleu A, Vinoy S, Holst JJ, Schaafsma G, Hendriks HF (2006) Effect of a high-protein breakfast on the postprandial ghrelin response. Am J Clin Nutr 83: $211-220$

Broglio F, Gianotti L, Destefanis S, Fassino S, Abbate Daga G, Mondelli V, Lanfranco F, Gottero C, Gauna C, Hofland L, Van der Lely AJ, Ghigo E (2004) The endocrine response to acute ghrelin administration is blunted in patients with anorexia nervosa, a ghrelin hypersecretory state. Clin Endocrinol (Oxford) 60: 592-599

Bruera E, Chadwick S, Cowan L, Drebit D, Hanson J, MacDonald N, van Konkelenberg Y (1986) Caloric intake assessment in advanced cancer patients: comparison of three methods. Cancer Treat Rep 70: $981-983$

Cassoni P, Allia E, Marrocco T, Ghe C, Ghigo E, Muccioli G, Papotti M (2006) Ghrelin and cortistatin in lung cancer: expression of peptides and related receptors in human primary tumors and in vitro effect on the H345 small cell carcinoma cell line. J Endocrinol Invest 29: $781-790$

Corbetta S, Peracchi M, Cappiello V, Lania A, Lauri E, Vago L, Beck-Peccoz P, Spada A (2003) Circulating ghrelin levels in patients with pancreatic and gastrointestinal neuroendocrine tumors: identification of one pancreatic ghrelinoma. J Clin Endocrinol Metab 88: 3117-3120

Date Y, Murakami N, Toshinai K, Matsukura S, Niijima A, Matsuo H, Kangawa K, Nakazato M (2002) The role of the gastric afferent vagal nerve in ghrelin-induced feeding and growth hormone secretion in rats. Gastroenterology 123: $1120-1128$ 
DeBoer MD, Zhu XX, Levasseur P, Meguid MM, Suzuki S, Inui A, Taylor JE, Halem HA, Dong JZ, Datta R, Culler MD, Marks DL (2007) Ghrelin treatment causes increased food intake and retention of lean body mass in a rat model of cancer cachexia. Endocrinology 148: 3004-3012

Depoortere I, Thijs T, Peeters T (2006) The contractile effect of the ghrelin receptor antagonist, D-Lys3-GHRP-6, in rat fundic strips is mediated through 5-HT receptors. Eur J Pharmacol 537: $160-165$

Dixit VD, Schaffer EM, Pyle RS, Collins GD, Sakthivel SK, Palaniappan R, Lillard Jr JW, Taub DD (2004) Ghrelin inhibits leptin- and activationinduced proinflammatory cytokine expression by human monocytes and T cells. J Clin Invest 114: 57-66

D'Onghia V, Leoncini R, Carli R, Santoro A, Giglioni S, Sorbellini F, Marzocca G, Bernini A, Campagna S, Marinello E, Vannoni D (2007) Circulating gastrin and ghrelin levels in patients with colorectal cancer: correlation with tumour stage, Helicobacter pylori infection and BMI. Biomed Pharmacother 61: 137-141

Druce MR, Neary NM, Small CJ, Milton J, Monteiro M, Patterson M, Ghatei MA, Bloom SR (2006) Subcutaneous administration of ghrelin stimulates energy intake in healthy lean human volunteers. Int $J$ Obes (London) 30: 293-296

Duxbury MS, Waseem T, Ito H, Robinson MK, Zinner MJ, Ashley SW, Whang EE (2003) Ghrelin promotes pancreatic adenocarcinoma cellular proliferation and invasiveness. Biochem Biophys Res Commun 309: 464-468

Ekeblad S, Nilsson B, Lejonklou MH, Johansson T, Stalberg P, Nilsson O, Ahlman H, Skogseid B (2006) Gastrointestinal stromal tumors express the orexigen ghrelin. Endocr Relat Cancer 13: 963-970

Enomoto M, Nagaya N, Uematsu M, Okumura $H$, Nakagawa $E$, Ono F, Hosoda H, Oya H, Kojima M, Kanmatsuse K, Kangawa K (2003) Cardiovascular and hormonal effects of subcutaneous administration of ghrelin, a novel growth hormone-releasing peptide, in healthy humans. Clin Sci (London) 105: $431-435$

Fearon KC, Voss AC, Hustead DS, Cancer Cachexia Study Group (2006) Definition of cancer cachexia: effect of weight loss, reduced food intake, and systemic inflammation on functional status and prognosis. Am J Clin Nutr 83: $1345-1350$

Garcia J, Boccia RV, Graham C, Kumor K, Polvino W (2007) A phase II randomized, placebo-controlled, double-blind study of the efficacy and safety of RC-1291 (RC) for the treatment of cancer cachexia. J Clin Oncol, 2007 ASCO Annu Meet Proc 25(Part I): 9133.22

Garcia JM, Garcia-Touza M, Hijazi RA, Taffet G, Epner D, Mann D, Smith RG, Cunningham GR, Marcelli M (2005) Active ghrelin levels and active to total ghrelin ratio in cancer-induced cachexia. J Clin Endocrinol Metab 90: $2920-2926$

Gilg S, Lutz TA (2006) The orexigenic effect of peripheral ghrelin differs between rats of different age and with different baseline food intake, and it may in part be mediated by the area postrema. Physiol Behav 87: $353-359$

Hanada T, Toshinai K, Date Y, Kajimura N, Tsukada T, Hayashi Y, Kangawa K, Nakazato M (2004) Upregulation of ghrelin expression in cachectic nude mice bearing human melanoma cells. Metabolism 53: $84-88$

Hanada T, Toshinai K, Kajimura N, Nara-Ashizawa N, Tsukada T, Hayashi Y, Osuye K, Kangawa K, Matsukura S, Nakazato M (2003) Anti-cachectic effect of ghrelin in nude mice bearing human melanoma cells. Biochem Biophys Res Commun 301: 275-279

Holst B, Schwartz TW (2006) Ghrelin receptor mutations - too little height and too much hunger. J Clin Invest 116: 637-641

Huang Q, Fan YZ, Ge BJ, Zhu Q, Tu ZY (2007) Circulating ghrelin in patients with gastric or colorectal cancer. Dig Dis Sci 52: 803-809

Hutton JL, Baracos VE, Wismer WV (2007) Chemosensory dysfunction is a primary factor in the evolution of declining nutritional status and quality of life in patients with advanced cancer. J Pain Symptom Manage 33: $156-165$

Inui A (2002) Cancer anorexia-cachexia syndrome: current issues in research and management. CA Cancer Clin 52: 72-91

Jaszberenyi M, Bujdoso E, Bagosi Z, Telegdy G (2006) Mediation of the behavioral, endocrine and thermoregulatory actions of ghrelin. Horm Behav 50: 266-273

Jeffery PL, Murray RE, Yeh AH, McNamara JF, Duncan RP, Francis GD, Herington AC, Chopin LK (2005) Expression and function of the ghrelin axis, including a novel preproghrelin isoform, in human breast cancer tissues and cell lines. Endocr Relat Cancer 12: 839-850

Kojima M, Hosoda H, Date Y, Nakazato M, Matsuo H, Kangawa K (1999) Ghrelin is a growth-hormone-releasing acylated peptide from stomach. Nature 402: 656-660
Kristenssson E, Sundqvist M, Astin M, Kjerling M, Mattsson H, Dornonville de la Cour C, Hakanson R, Lindstrom E (2006) Acute psychological stress raises plasma ghrelin in the rat. Regul Pept 134: 114-117

le Roux CW, Neary NM, Halsey TJ, Small CJ, Martinez-Isla AM, Ghatei MA, Theodorou NA, Bloom SR (2005) Ghrelin does not stimulate food intake in patients with surgical procedures involving vagotomy. J Clin Endocrinol Metab 90: 4521 - 4524

Lebenthal Y, Gat-Yablonski G, Shtaif B, Padoa A, Phillip M, Lazar L (2006) Effect of sex hormone administration on circulating ghrelin levels in peripubertal children. J Clin Endocrinol Metab 91: 328-331

Lejeune MP, Westerterp KR, Adam TC, Luscombe-Marsh ND, Westerterp-Plantenga MS (2006) Ghrelin and glucagon-like peptide 1 concentrations, 24-h satiety, and energy and substrate metabolism during a high-protein diet and measured in a respiration chamber. $\mathrm{Am}$ J Clin Nutr 83: 89-94

Levin F, Edholm T, Schmidt PT, Gryback P, Jacobsson H, Degerblad M, Hoybye C, Holst JJ, Rehfeld JF, Hellstrom PM, Naslund E (2006) Ghrelin stimulates gastric emptying and hunger in normal-weight humans. J Clin Endocrinol Metab 91: 3296-3302

Liu YL, Malik NM, Sanger GJ, Andrews PL (2006) Ghrelin alleviates cancer chemotherapy-associated dyspepsia in rodents. Cancer Chemother Pharmacol 58: $326-333$

Mellemkjaer L, Emborg C, Gridley G, Munk-Jorgensen P, Johansen C, Tjonneland A, Kjaer SK, Olsen JH (2001) Anorexia nervosa and cancer risk. Cancer Causes Control 12: 173-177

Miljic D, Pekic S, Djurovic M, Doknic M, Milic N, Casanueva FF, Ghatei M, Popovic V (2006) Ghrelin has partial or no effect on appetite, growth hormone, prolactin, and cortisol release in patients with anorexia nervosa. J Clin Endocrinol Metab 91: $1491-1495$

Mottershead M, Karteris E, Barclay JY, Suortamo S, Newbold M, Randeva $\mathrm{H}$, Nwokolo CU (2007) Immunohistochemical and quantitative mRNA assessment of ghrelin expression in gastric and oesophageal adenocarcinoma. J Clin Pathol 60: 405-409

Mundinger TO, Cummings DE, Taborsky Jr GJ (2006) Direct stimulation of ghrelin secretion by sympathetic nerves. Endocrinology 147: 2893-2901

Murray CD, Martin NM, Patterson M, Taylor SA, Ghatei MA, Kamm MA, Johnston C, Bloom SR, Emmanuel AV (2005) Ghrelin enhances gastric emptying in diabetic gastroparesis: a double blind, placebo controlled, crossover study. Gut 54: 1693-1698

Nagaya N, Itoh T, Murakami S, Oya H, Uematsu M, Miyatake K, Kangawa K (2005) Treatment of cachexia with ghrelin in patients with COPD. Chest 128: 1187-1193

Nagaya $N$, Kojima $M$, Uematsu M, Yamagishi M, Hosoda H, Oya $H$, Hayashi Y, Kangawa K (2001a) Hemodynamic and hormonal effects of human ghrelin in healthy volunteers. Am J Physiol Regul Integr Comp Physiol 280: R1483-R1487

Nagaya N, Miyatake K, Uematsu M, Oya H, Shimizu W, Hosoda H, Kojima M, Nakanishi N, Mori H, Kangawa K (2001b) Hemodynamic, renal, and hormonal effects of ghrelin infusion in patients with chronic heart failure. J Clin Endocrinol Metab 86: 5854-5859

Nagaya N, Moriya J, Yasumura Y, Uematsu M, Ono F, Shimizu W, Ueno K, Kitakaze M, Miyatake K, Kangawa K (2004) Effects of ghrelin administration on left ventricular function, exercise capacity, and muscle wasting in patients with chronic heart failure. Circulation 110: $3674-3679$

Neary NM, Small CJ, Wren AM, Lee JL, Druce MR, Palmieri C, Frost GS, Ghatei MA, Coombes RC, Bloom SR (2004) Ghrelin increases energy intake in cancer patients with impaired appetite: acute, randomized, placebo-controlled trial. J Clin Endocrinol Metab 89: 2832 - 2836

Ohno T, Kamiyama Y, Aihara R, Nakabayashi T, Mochiki E, Asao T, Kuwano H (2006) Ghrelin does not stimulate gastrointestinal motility and gastric emptying: an experimental study of conscious dogs. Neurogastroenterol Motil 18: 129-135

Omlin AG, Strasser F (2007) Secondary causes of cancer-related anorexia: recognition in daily practice by a novel checklist, a pilot study. J Clin Oncol, 2007 ASCO Annu Meet Proc 25(Part I): 9058

Rudd JA, Ngan MP, Wai MK, King AG, Witherington J, Andrews PL, Sanger GJ (2006) Anti-emetic activity of ghrelin in ferrets exposed to the cytotoxic anti-cancer agent cisplatin. Neurosci Lett 392: 79-83

Schmid DA, Held K, Ising M, Uhr M, Weikel JC, Steiger A (2005) Ghrelin stimulates appetite, imagination of food, GH, ACTH, and cortisol, but does not affect leptin in normal controls. Neuropsychopharmacology 30: 1187-1192

Shimizu $\mathrm{Y}$, Nagaya $\mathrm{N}$, Isobe $\mathrm{T}$, Imazu $\mathrm{M}$, Okumura $\mathrm{H}$, Hosoda $\mathrm{H}$, Kojima M, Kangawa K, Kohno N (2003) Increased plasma ghrelin level in lung cancer cachexia. Clin Cancer Res 9: 774-778 
Smith RG (2005) Development of growth hormone secretagogues. Endocr Rev 26: $346-360$

Strasser F, Palmer JL, Schover LR, Yusuf SW, Pisters K, VassilopoulouSellin R, DeGracia B, Willey JS, Bruera E (2006) The impact of hypogonadism and autonomic dysfunction on fatigue, emotional function, and sexual desire in male patients with advanced cancer: a pilot study. Cancer 107: 2949-2957

Stromgren AS, Sjogren P, Goldschmidt D, Petersen MA, Pedersen L, Groenvold M (2006) Symptom priority and course of symptomatology in specialized palliative care. J Pain Symptom Manage 31: 199-206

Tack J, Depoortere I, Bisschops R, Delporte C, Coulie B, Meulemans A, Janssens J, Peeters T (2006) Influence of ghrelin on interdigestive gastrointestinal motility in humans. Gut 55: 327-333

Theander-Carrillo C, Wiedmer P, Cettour-Rose P, Nogueiras R, Perez-Tilve D, Pfluger P, Castaneda TR, Muzzin P, Schurmann A, Szanto I, Tschop MH, Rohner-Jeanrenaud F (2006) Ghrelin action in the brain controls adipocyte metabolism. J Clin Invest 116: $1983-1993$

Tsolakis AV, Portela-Gomes GM, Stridsberg M, Grimelius L, Sundin A, Eriksson BK, Oberg KE, Janson ET (2004) Malignant gastric ghrelinoma with hyperghrelinemia. J Clin Endocrinol Metab 89: 3739-3744
Wang W, Andersson M, Iresjo BM, Lonnroth C, Lundholm K (2006) Effects of ghrelin on anorexia in tumor-bearing mice with eicosanoid-related cachexia. Int J Oncol 28: $1393-1400$

Wei W, Qi X, Reed J, Ceci J, Wang HQ, Wang G, Englander EW, Greeley Jr $\mathrm{GH}$ (2006) Effect of chronic hyperghrelinemia on ingestive action of ghrelin. Am J Physiol Regul Integr Comp Physiol 290: R803-R808

Wolf I, Sadetzki S, Kanety H, Kundel Y, Pariente C, Epstein N, Oberman B Catane R, Kaufman B, Shimon I (2006) Adiponectin, ghrelin, and leptin in cancer cachexia in breast and colon cancer patients. Cancer 106: $966-973$

Wren AM, Seal LJ, Cohen MA, Brynes AE, Frost GS, Murphy KG, Dhillo WS, Ghatei MA, Bloom SR (2001) Ghrelin enhances appetite and increases food intake in humans. J Clin Endocrinol Metab 86: 5992

Yavuzsen T, Davis MP, Walsh D, LeGrand S, Lagman R (2005) Systematic review of the treatment of cancer-associated anorexia and weight loss. $J$ Clin Oncol 23: $8500-8511$

Yeh AH, Jeffery PL, Duncan RP, Herington AC, Chopin LK (2005) Ghrelin and a novel preproghrelin isoform are highly expressed in prostate cancer and ghrelin activates mitogen-activated protein kinase in prostate cancer. Clin Cancer Res 11: 8295-8303 